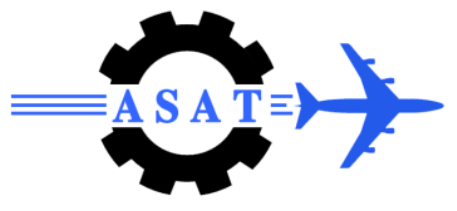

\title{
An ILC-Based Adaptive PI Controller for Two-Link Robot Manipulator: Minimum Entropy Approach
}

\author{
Z. Skaf*, M. Ashry ${ }^{\dagger}$ and A. Al-Bayati ${ }^{\ddagger}$
}

\begin{abstract}
In this paper, a new algorithm for an adaptive Proportional-Integrator (PI) controller for nonlinear systems subject to stochastic non-Gaussian disturbance is studied. The minimum entropy control is applied to decrease the closed-loop tracking error under an iterative learning control (ILC) basis. The key issue here is to divide the control horizon into a number of equal time intervals called batches. Within each interval there are a fixed number of sample points. The design procedure is divided into two main algorithms, within each batch and between any two adjacent batches. A D-type ILC laws are employed to tune the PI controller coefficients between two adjacent batches. However, within each batch, the PI coefficients are fixed. A sufficient condition has been established to guarantee the stability of the closed-loop system. An illustrated example of two-link is included to demonstrate the use of control algorithm, and satisfactory results have been obtained.
\end{abstract}

Keywords: Entropy, nonlinear systems, iterative control.

\section{Introduction}

Paper Research into controllers design for stochastic systems has been regarded as an important aspect over the past decades. This is mainly due to the fact that a large class of physical systems has random inputs, time-delays, uncertainties and noises. Therefore, an ideal control design should be such that the systems preferably attenuate the stochastic behavior, or the effects caused by randomness are minimized effectively. Under the assumption that the random variables or the noise in the stochastic system are subject to Gaussian processes, the tracking error has been used to represent the closed-loop performance. The statistics of the tracking error thus characterize the performance of the controller. The first approach on the controller design has been focused on the mean and variance control [1], where the uncertainties of the closed-loop stochastic systems are minimized. Later on, linear optimal control [2] and linear quadratic martingale control [3] was developed. Further research has been performed for optimal stochastic control, optimal adaptive predictive control for nonlinear stochastic systems and stochastic adaptive control strategies presented in [4], [5], and [6], respectively. Also, sliding mode control problem for a class of linear continuous-time systems with Markovian jump parameters has been solved in [7], and robust fuzzy control for uncertain Markovian stochastic systems has been developed by [8]. More recently, robust $H$ control of uncertain stochastic nonlinear systems [9], and adaptive output feedback controller

\footnotetext{
* Control Systems Center, University of Manchester, Manchester, M60 1QD, UK, zakwan.skaf@manchester.ac.uk

Egyptian Armed Forces, Egypt, mmaashry@yahoo.com

$\$$ PhD Student, Control Systems Center, University of Manchester, Manchester, M60 1QD, UK, ahmad al-bayati@ postgrad.manchester.ac.uk
} 
for stochastic nonlinear system [10] have been introduced. On the other hand, when the random variables or the noise in the stochastic system are subjected to non-Gaussian processes, it has been shown that mean and variance are insufficient to characterize the stochastic properties. This is because the spread area of a non-symmetric distribution cannot be accurately described by the variance only. Therefore, a new method for randomness measure, called the entropy of the tracking error of the closed-loop system, should be employed for the closed-loop control design of the non-Gaussian stochastic systems. The entropy has a significant advantage in dealing with non-Gaussian system. This is because the entropy provides a general description of the uncertainties of stochastic systems without constraints of certain distributions. Moreover, it has been proved that the minimum entropy algorithm is similar to the minimum variance algorithm when the noise of the stochastic system is subjected to Gaussian processes [11], and [12]. The entropy has been used in different definitions. Initially, the entropy has been presented as a measure of the uncertainty about its actual structure in both continuous and discrete time [13]. Later on, a new definition has been presented in [14] by considering the exponential nature of the information gain function. Also, the entropy has been represented as an average information content in a given probability density function [15]. In fact, entropy concept has been applied widely in the design of control algorithms for stochastic systems. Among various existing approaches is the work of Wang [11], where the entropy has been applied to characterize and minimize the closed loop randomness for general stochastic systems subjected to arbitrary bounded random inputs. The control input is formulated which minimizes the output entropy and guarantee the local stability of the closed-loop system. Later on, [12] have developed a control algorithm for the control of the output mean values and the minimisation of the closed-loop entropy for nonlinear affine and non-Gaussian stochastic systems. Moreover, [16] have established a novel controller design for a linear time-invariant stochastic system subjected to a bounded random input by using Youla parameterization to formulate the structure of the controller and to guarantee the closed loop stability. Also, a minimum entropy control problem has been solved for nonlinear ARMA systems over a communication network with a stochastic delay in the communication channels, where the probability density function of the tracking error has been estimated by using a neural network [17]. In the most recent work by [18], an adaptive control for general nonlinear and non-Gaussian unknown stochastic systems has been established. For this purpose, the control horizon is divided into a number of equal time domain intervals called batches. Within each interval there are a fixed number of sample points. The design procedure has been divided into two main algorithms, within each batch and between any two adjacent batches. The minimum entropy control has been applied to decrease the closed-loop randomness of the output under an iterative learning control basis. The neural network dynamics has been constructed to perform both modeling and control of the plant.

The paper is organized as follows. Section 2 briefly describes the problem. Section 3 presents the controller algorithm and develops a parameters tuning method. Section 4 presents the sufficient condition to guarantee the stability of the closed-loop system. Section 5 illustrates the application of the design methodology to a two-link manipulator problem. Finally, the concluding remarks are proposed in section 6 .

\section{Problem Formulation}

In this work the following nonlinear stochastic model between the output and input will be considered

$$
\begin{aligned}
\mathrm{x}(\mathrm{i}+1) & =\mathrm{Ax}(\mathrm{i})+\mathrm{Bu}(\mathrm{i})+\mathrm{g}(\mathrm{x}(\mathrm{i}))+\mathrm{d}(\mathrm{i}) \\
\mathrm{y}(\mathrm{i}) & =\mathrm{Cx}(\mathrm{i})
\end{aligned}
$$

where $x(i) \in R^{n}$ is the state vector, $u(i) \in R^{r}$ is the measurable input vector and $y(i) \in R^{p}$ is the measurable output vector. Moreover, $A, B$ and $C$ represent the known parametric matrices of 
the dynamic part of the system. $d(i)$ stands for the bounded non-Gaussian random noise. $g(x(i))$ is a nonlinear vector function that stands for the nonlinear dynamics of the model and is supposed to satisfy the following norm condition

$\left\|g\left(x_{1}(i)\right)-g\left(x_{2}(i)\right)\right\| \leq\left\|U\left(x_{1}(i)-x_{2}(i)\right)\right\|$

for any $x_{1}(i)$ and $x_{2}(i)$, where $U$ is a known matrix. Denoting the desire system output as $r(i)$ the tracking error can be expressed as

$e(i)=r(i)-y(i)$

which is a non-Gaussian random process due to the affect of the non-Gaussian disturbance on the closed-loop performance. Theoretically, it is expected that such a randomness or uncertainty is minimized by the controller function. In other words, the purpose of the PI controller design in this work is to establish a control signal so that the randomness or uncertainty in the system output and closed-loop tracking error is minimized. The controller coefficients will be trained by an ILC tuning mechanism to minimize the entropy of the closed-loop tracking error. For this purpose, the well-known Renyis entropy measure for a random error (e) can be expressed as follows

$$
H(e)=\frac{1}{1-\alpha} \log \left(\int \gamma^{\alpha}(e) d e\right)
$$

where $\gamma(e)$ presents the Probability Density Function (PDF) of the random error. The following Kernel density estimation method is used to estimate the tracking error probability distribution function [15].

$$
\gamma(e) \approx \hat{\gamma}(e)=\frac{1}{N} \sum_{i=1}^{N} K_{\sigma}\left(e-e_{i}\right)
$$

where $K_{\sigma}$ is a real, symmetrical Kernel function with the specifications stated in [15]. $\sigma>0$ is the order of Renyis quadratic entropy. The chosen Kernel function in this paper is expressed as follows:

$$
K_{\sigma}(e)=\frac{1}{\sqrt{2 \pi}} \exp \left(\frac{-e^{2}}{2 \sigma^{2}}\right)
$$

The choice of the Kernel function is actually dependent on the level of smoothness the designer expects from the PDF estimation. The ILC-based controller tuning objective function can be written as follows:

$$
H(e)=\frac{1}{1-\alpha} \log \left(V_{R a}(e)\right)
$$

where $V_{R a}(e)$ is usually called the Information Potential (IP), and can be further expressed as

$$
V_{R a}(e)=\frac{1}{N^{\alpha}} \sum_{i=1}^{N}\left[\sum_{j=1}^{N} K_{\sigma}\left(e_{i}-e_{j}\right)\right]^{\alpha}
$$

\section{Controller Algorithm Design}

PI controllers are widely used in both theoretical studies and practical applications of industrial control over the last five decades [19]. The popularity of PI controller can be attributed to its robust performance in a wide range of operating conditions and to its very simple structure. As discussed before, the aim of the PI controller design is to minimize the randomness of the tracking error, so that the entropy of the tracking error can be minimized. For this purpose, the control horizon will be divided into a number of equal time-domain intervals called batches as $\left\{T^{1}, T^{2}, T^{3}, \ldots T^{k}, ..\right\}$. Within each interval there are a fixed number of sample points $\{1,2 \ldots, N\}$, where $N$ is sampling numbers in a batch. In each batch a number of sampled tracking errors can be collected. Between any two adjacent batches, the PI coefficients are tuned by ILC-based method to minimize the tracking error entropy (Fig. 1). 
The ILC tuning mechanism of PI coefficients needs to go on at the beginning of the $\mathrm{k}$ batches until the PI coefficients cannot be further tuned.

A generalized PI controller with tunable coefficients is considered as adaptive controller in this work as follows

$$
\begin{aligned}
& \zeta(i)=\zeta(i-1)+T_{s} e(i-1) \\
& u(i)=K_{P} e(i)+K_{I} \zeta(i)
\end{aligned}
$$

where $T_{S}$ is the sampling time.

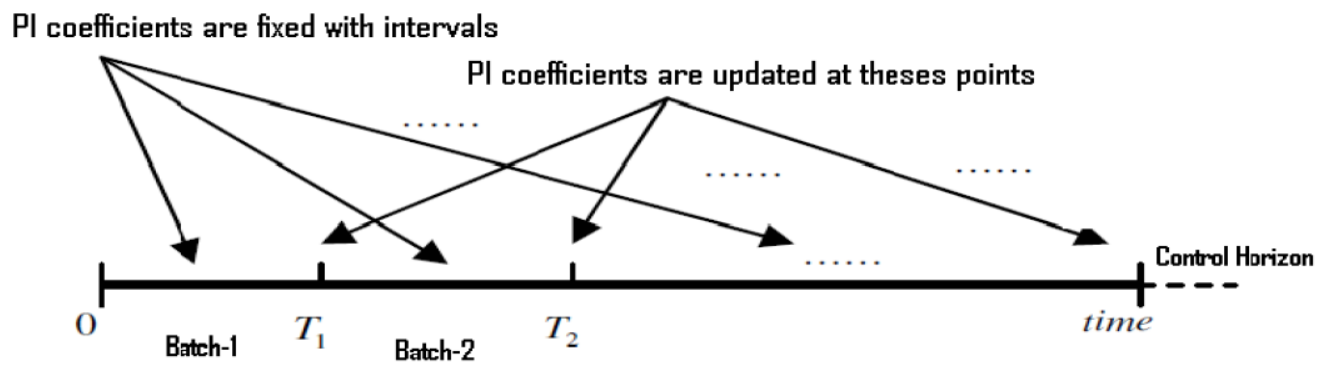

Fig. 1. PI tuning procedure.

Assume that the current instant is at the beginning of the interval $T^{k}$, the system tracking error within this interval should have the following sample values. $\left\{e_{1}^{k}, e_{2}^{k}, e_{3}^{k}, \ldots . . e_{N}^{k}\right\}$.Within this interval the sampled tracking error can be used to measure the entropy and construct the PDF. Therefore, the entropy of sample $\left\{e_{1}^{k-1}, e_{2}^{k-1}, e_{3}^{k-1}, \ldots . . e_{N}^{k-1}\right\}$ in the interval $T^{k-1}$ can be expressed as follows:

$$
H^{k-1}(e)=\frac{1}{1-\alpha} \log \left\{\frac{1}{N^{\alpha}} \sum_{i=1}^{N}\left[\sum_{j=1}^{N} K_{\sigma}\left(e_{i}^{k-1}-e_{j}^{k-1}\right)\right]^{\alpha-1}\right\}
$$

and the information potential for $\left\{e_{1}^{k-1}, e_{2}^{k-1}, e_{3}^{k-1}, \ldots . . e_{N}^{k-1}\right\}$ in the interval $T^{k-1}$ can be expressed according to the following equation

$$
V_{R a}^{k-1}(e)=\frac{1}{N^{\alpha}} \sum_{i=1}^{N}\left[\sum_{j=1}^{N} K_{\sigma}\left(e_{i}^{k-1}-e_{j}^{k-1}\right)\right]^{\alpha-1}
$$

By substituting (11) into (10), the entropy can be obtained as follows:

$$
H^{k-1}(e)=\frac{1}{1-\alpha} \log V_{R a}^{k-1}(e)
$$

The solution to tune the PI controller coefficients between adjacent batches can be solved by the following D-type ILC law which can be only considered as a local optimal solution

$$
\begin{aligned}
& K_{P}(k)=K_{P}(k-1)-\lambda_{P} \frac{\partial H^{k-1}(e)}{\partial K_{P}^{k-1}} \\
& K_{I}(k)=K_{I}(k-1)-\lambda_{I} \frac{\partial H^{k-1}(e)}{\partial K_{I}^{k-1}}
\end{aligned}
$$

where $\lambda_{P}$ and $\lambda_{I}$ are the ILC learning rates chosen so that the iterative control law is convergent [20]. $K_{P}(k)$ and $K_{I}(k)$ are the PI controller coefficients within the $K^{\text {th }}$ batch. In ILC, the PI coefficients in the $K^{\text {th }}$ batch is based on the PI coefficients in the $(K-1)^{\text {th }}$ batch and a correcting function, which is basically dependent on the gradient of closed-loop entropy tracking error of PI coefficients in the $(K-1)^{t h}$ batch. The $K^{\text {th }}$ component of $\partial H^{k-1}(e) / \partial K_{P}{ }^{k-1}$, $\partial H^{k-1}(e) / \partial K_{I}^{k-1}$ can be approximated as follows:

$\Delta K_{P}=K_{p}(k-1)-K_{P}(k-2)$

$\Delta K_{I}=K_{I}(k-1)-K_{I}(k-2)$ 
To guarantee the convergence of the above proposed ILC algorithm, the learning rate of PI coefficients should be decreased batch-by-batch. In other words, the entropy of closed-loop tracking error should be decreased batch-by batch. This would be equivalent to

$H^{k}(e(i))<H^{k-1}(e(i))$

Since $\alpha>0$ then inequality (15) can be re-written as

$\log V_{R a}^{k-1}(e(i))<\log V_{R a}^{k}(e(i))$

which means that

$\log \left(\frac{V_{R a}^{k-1}(e(i))}{V_{R a}^{k}(e(i))}\right)<0$

which is equivalent to the following

$\frac{V_{R a}^{k-1}(e(i))}{V_{R a}^{k}(e(i))}<1$

Since the IP is nonnegative, inequality (16) would mean

$\Delta V_{R a}=V_{R a}^{k}(e(i))-V_{R a}^{k-1}(e(i))>0$

\section{Closed Loop Stability}

In this section, the stability of the closed-loop system given by (1) and (11) will be considered. Corresponding to equations (10), (11), and (2), the closed-loop system equation within the $K^{\text {th }}$ batch can be written as

$$
M_{k}(i+1)=\bar{A} M_{k}(i)+\bar{B} r+\bar{F} g\left(M_{k}(i)\right)
$$

where

$$
M_{k}(i)=\left[\begin{array}{c}
e_{k}(i) \\
\zeta(i)
\end{array}\right], \bar{A}=\left[\begin{array}{cc}
A-B K_{P} & -B K_{I} \\
T_{s} I & I
\end{array}\right], \bar{B}=\left[\begin{array}{l}
I-A \\
0
\end{array}\right], \bar{F}=\left[\begin{array}{ll}
I & 0 \\
0 & 0
\end{array}\right], g\left(M_{k}(i)\right)=\left[\begin{array}{l}
g(x(i)) \\
0
\end{array}\right]
$$

To prove the stability of the closed loop system, let us consider the following Lyapunov candidate

$$
V\left(M_{k}(i), i\right)=M_{k}^{T}(i) P^{-1} M_{k}(i)+\lambda^{2} \sum_{j=1}^{i-1}\left[\left\|\bar{U} M_{k}(i)\right\|^{2}-\left\|g\left(M_{k}(i)\right)\right\|\right]
$$

where $\bar{U}=\left[\begin{array}{ll}U & 0 \\ 0 & 0\end{array}\right]$

Differentiating equation (22) over the time gives

$$
\begin{aligned}
& \Delta V(i)=V\left(M_{k}(i+1), i+1\right)-V\left(M_{k}(i), i\right) \\
& \Delta V(i)=\bar{S}_{k}^{T}(i) N_{K} \bar{S}_{k}(i)+2 \bar{S}_{k}{ }^{T}(i) \bar{A}^{T} P^{-1} \bar{B} r+2 g^{T}\left(M_{K}(i)\right) \bar{F}^{T} P^{-1} \bar{B} r+r^{T} \bar{B}^{T} P^{-1} \bar{B} r<0
\end{aligned}
$$

where

$$
N_{K}=\left[\begin{array}{lr}
\bar{A}^{T} P^{-1} \bar{A}-P^{-1}+\lambda^{2} \bar{U}^{T} \bar{U} & \bar{A}^{T} P^{-1} \bar{F} \\
\bar{F}^{T} P^{-T} \bar{A} & \bar{F}^{T} P^{-T} \bar{A}-\lambda^{2} I
\end{array}\right]
$$

Using the well known Schur complement formula, (24) can be further reduced to

$$
N_{1, K}=\left[\begin{array}{lcl}
-P^{-1}+\lambda^{2} \bar{U}^{T} \bar{U} & 0 & \bar{A}^{T} \\
0 & -\lambda^{2} I & \bar{F}^{T} \\
\bar{A} & 0 & -P
\end{array}\right]
$$

By pre-multiplying $N_{1, K}$ by $\operatorname{diag}\left(P_{k}^{T}, I, I\right)$ and post multiplying it by $\operatorname{diag}\left(P_{k}, I, I\right)$ and also applying Schur complement formula, the necessary condition for stability will be as follows 
$N_{2, K}=\left[\begin{array}{lrcc}-P & 0 & P^{T} \bar{A}^{T} & \lambda P \bar{U}^{T} \\ 0 & -\lambda^{2} I & \bar{F}^{T} & 0 \\ \bar{A} P & \bar{F} & -P & 0 \\ \lambda \bar{U} P & 0 & 0 & -I\end{array}\right]$

If (26) holds, a positive scalar $\delta$ exists so that $N_{k} \leq-\delta I$. Along with (20) it can be verified that

$\Delta V(i) \leq-\delta\left\|\bar{S}_{k}\right\|^{2}+2\left\|\bar{A}_{k}{ }^{T} P^{-1} \bar{B}_{k} r\right\| \cdot\left\|\bar{S}_{k}\right\|+2 g^{T}\left(M_{k}(i)\right) \bar{F}^{T} P^{-1} \bar{B}_{k} r+r^{T} \bar{B}^{T} P^{-1} \bar{B} r$

It is obvious that the right-hand side of inequality (27) is a second degree polynomial with respect to $\bar{S}_{k}(i)$. It can be shown that $\Delta V(i) \leq 0$ holds if

$\bar{S}_{k}(i) \geq \delta^{-1}\left(\left\|\bar{A}_{k}^{T} P^{-1} \bar{B}_{k} r\right\|+\sqrt{\left\|\bar{A}_{k}^{T} P^{-1} \bar{B}_{k} r\right\|^{2}+\delta\left(c_{1}+c_{2}\right)}\right)$

where

$c_{1}=2 g^{T}\left(M_{k}(i)\right) \bar{F}^{T} P^{-1} \overline{B_{k}} r, \quad c_{2}=r^{T} B^{T} P^{-1} B r$

This means that the stability of the closed-loop system can be checked.

\section{Simulation Results}

Using the Lagrange Euler formulation, the dynamic equation of a $n$-joint of an ideal rigid robot arm can be expressed with the equation of motion given by [21]

$M(q) \ddot{q}+c(q, \dot{q}) \dot{q}+g(q)=u$

where $q \in R^{n}$ is the joint angular position vector of the robot manipulator; $u \in R^{n}$ is the applied joint torques; $M(q) \in R^{m \times n}$ is the inertia matrix; $c(q, \dot{q}) \in R^{m \times n}$ is the effect of Coriolis and centrifugal forces; $g(q) \in R^{n}$ is the gravitational torques.

In this work, a two-link robot manipulator as shown in Fig. 2. is considered. The parameter matrices are as follows [22]:

$$
\begin{aligned}
& M(q)=\left[\begin{array}{cc}
\theta_{1}+\theta_{2}+2 \theta_{3} \cos \left(q_{2}\right) & \theta_{2}+2 \theta_{3} \cos \left(q_{2}\right) \\
\theta_{2}+2 \theta_{3} \cos \left(q_{2}\right) & \theta_{2}
\end{array}\right], c(q, \dot{q})=\left[\begin{array}{cc}
-\theta_{3} \sin \left(q_{2}\right) \dot{q}_{2} & -\theta_{3} \sin \left(q_{2}\right)\left(\dot{q}_{1}+\dot{q}_{2}\right) \\
\theta_{3} \sin \left(q_{2}\right) \dot{q}_{1} & 0
\end{array}\right] \\
& g(q)=\left[\begin{array}{c}
g\left(\theta_{4}+\theta_{5}\right) \cos \left(q_{1}\right)+g \theta_{6} \cos \left(q_{1}+q_{2}\right) \\
g \theta_{6} \cos \left(q_{1}+q_{2}\right)
\end{array}\right]
\end{aligned}
$$

where $g$ is the gravitational acceleration and

$$
\theta_{1}=m_{1} l_{c 1}^{2}+m_{2} l_{c 2}^{2}+I_{1}, \theta_{2}=m_{2} l_{c 2}^{2}+I_{2}, \theta_{3}=m_{2} l_{c 1} l_{c 2}, \theta_{4}=m_{1} l_{c 1}, \theta_{5}=m_{1} l_{1}, \theta_{6}=m_{2} l_{c 2}
$$

The numerical values for the two-link robot are presented in Table 1. The desired trajectories are:

$q_{d}=\left[\begin{array}{l}q_{1} \\ q_{2}\end{array}\right]=\left[\begin{array}{l}3.14^{*}(1-\exp (-0.5 t) \\ 3.14^{*}(1-\exp (-0.5 t)\end{array}\right]$

Table 1 The parameter values of two-link robot

\begin{tabular}{|c|c|c|c}
\hline \hline$m_{1}=m_{2}$ & $l_{c 1}=l_{c 2}$ & $l_{1}=l_{2}$ & $I_{1}=I_{2}$ \\
\hline $10 \mathrm{Kg}$ & $0.5 \mathrm{~m}$ & $1 \mathrm{~m}$ & $1 \mathrm{Kg} \cdot \mathrm{m}^{2}$ \\
\hline \hline
\end{tabular}

Assuming the following initial values $[1 ; 1 ; 0 ; 0]$. Fig. 3 shows that the proposed algorithm works as expected by comparing the shape of error PDF in the first batch and the last iteration. Moreover, the 3-D mesh of the PDF of the closed loop tracking error further confirms that the closed loop tracking error tends to be a Gaussian-like shape, as shown in 
Fig. 4. Although some small fluctuations can be seen in the entropy variations, the overall trend of the closed-loop error entropy suggests a minimum has been achieved. Fig. 5 illustrates how the closed-loop error entropy is minimised along with the batches.

By choosing the ILC learning rates $\lambda_{P}(1)=2$ and $\lambda_{I}(1)=5$. Figs. 6 and 7 show the ILC learning rates converge throughout the batches. Also, the ILC-based tuning algorithm sets PI controller to $K_{P}=169.3 I$ and $K_{I}=79.5 I$ in the last batch as shown in Figs 8 and 9. From simulated results which are shown in Figs. 10 and 11, it can be seen that both $q_{1}$ and $q_{2}$ converge to the desired trajectories.

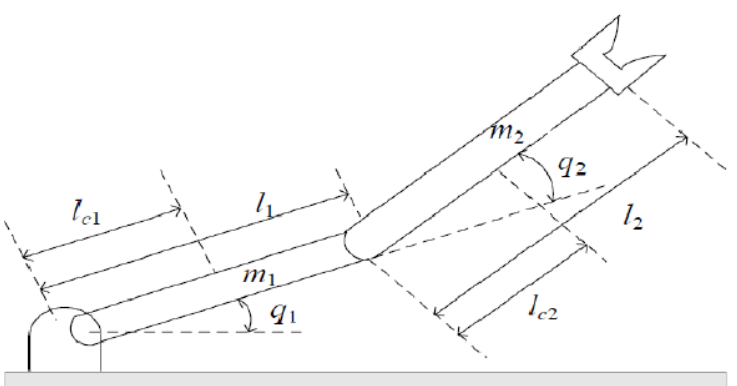

Fig. 2. Two link robot manipulator.

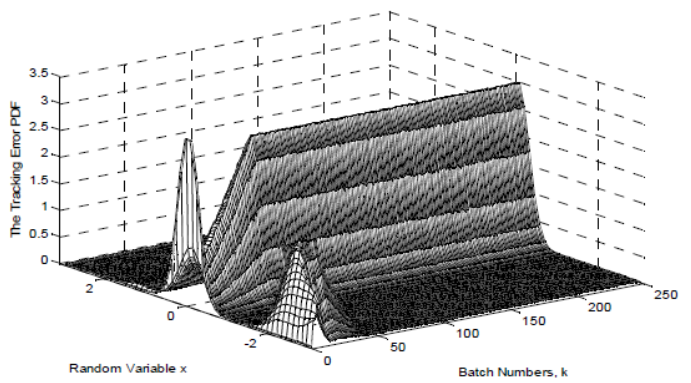

Fig. 4. The 3D mesh of the tracking error.

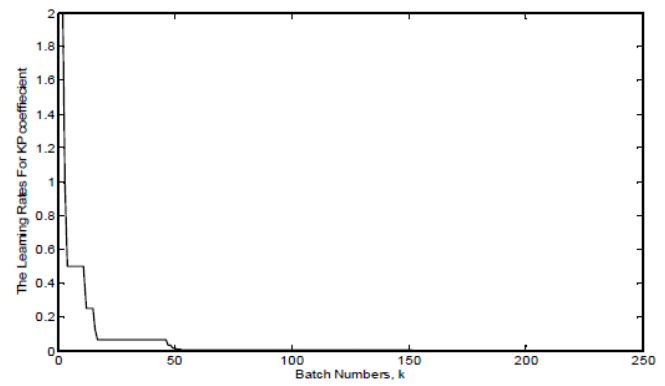

Fig. 6. $\lambda_{P}$ converge.

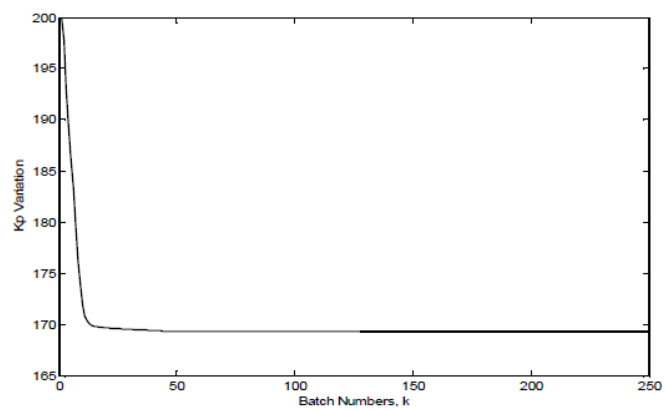

Fig. 8. $K_{P}$ variation.

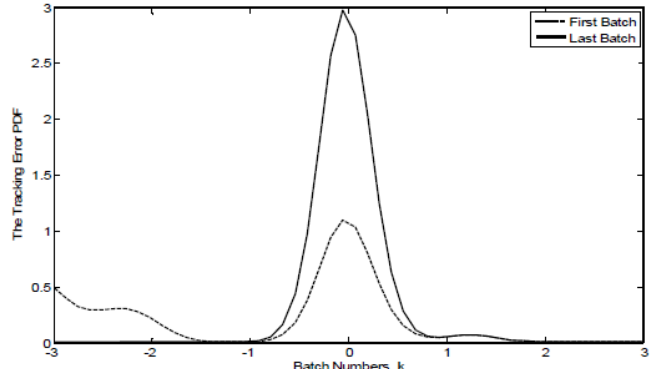

Fig. 3. Error PDF for first and last batch.

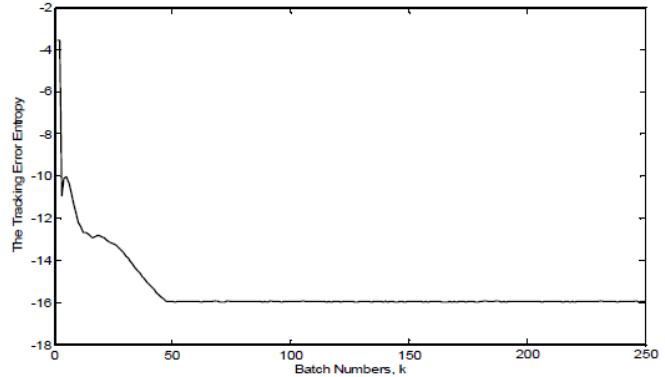

Fig. 5. Error entropy along with batches.

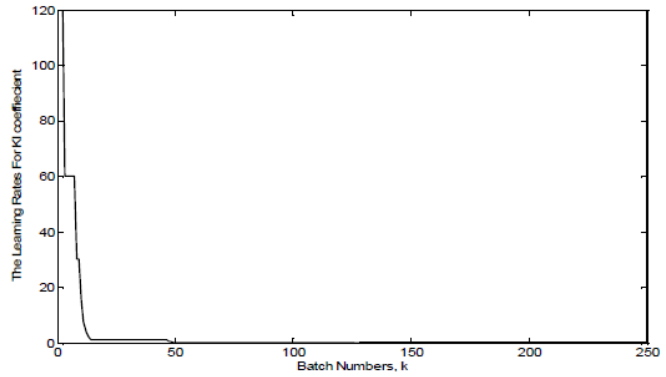

Fig. 7. $\lambda_{I}$ converge.

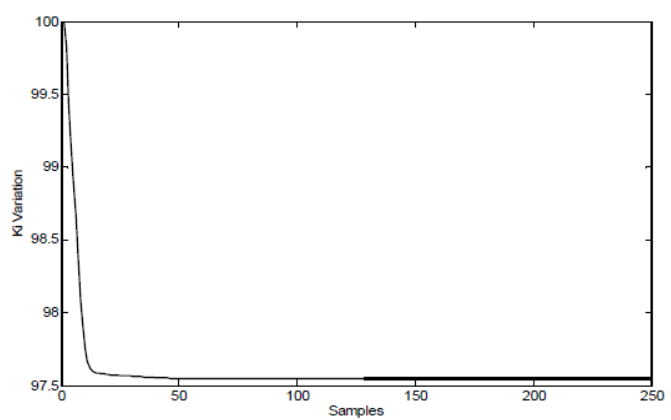

Fig. 9. $K_{I}$ variation. 


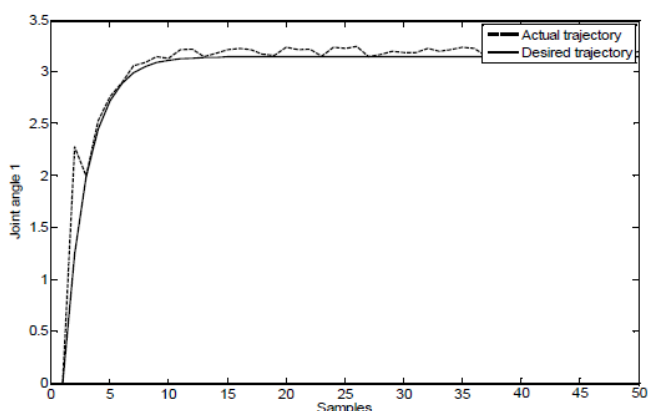

Fig. 10. Actual and desired bath of link 1.

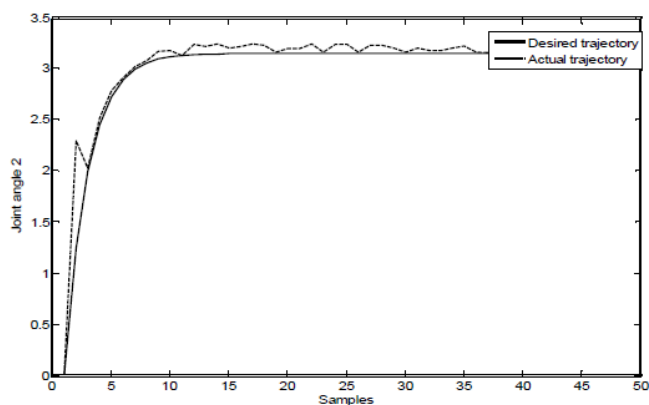

Fig. 11. Actual and desired bath of link 2.

\section{Conclusion}

The idea of ILC-based adaptive minimum entropy control was applied to nonlinear stochastic systems subject to non-Gaussian disturbance in this paper. A modified PI controller was developed for the purpose of minimum entropy. In this algorithm, the whole control horizon is divided into a number of equal time intervals, called batches. Within each interval, there are a fixed number of sample points. Within each batch, the minimum entropy control is realized, whilst between any two adjacent batches, the coefficients of the PI controller are tuned. A simple ILC law, namely the D-Type ILC, was applied for the tuning between any two adjacent batches, and a condition for the ILC convergence was established. The closed-loop stability of the system was discussed. An illustrated example of two-link robot manipulator was included to demonstrate the effectiveness of the proposed control algorithm. The simulation results demonstrate that the randomness of the closed-loop tracking error is minimized batch by batch, and the ILC learning rates converge throughout the batches. Moreover, the error PDF is a Gaussian-like shape in the last batch.

\section{References}

[1] Astrom. K., Introduction to Stochastic Control Theory, New York: Academic Press, 1970.

[2] Anderson. B. and Moore. J., Linear Optimal Control, Englewood Cliffs, NJ: Prentice Hall, 1971.

[3] Solo, V., "Stochastic adaptive control and martingale limit theory", Automatic Control, IEEE Transactions on, " vol. 35, no. 1, 1990, pp. $66-71$.

[4] Yaz, E., "Optimal stochastic control for performance and stability robustness”, Automatic Control, IEEE Transactions on, " vol. 38, no. 5, 1993, pp. 757 -760.

[5] Filatoy, N. and Unbehausen, H.," Adaptive predictive control policy for nonlinear stochastic systems”, Automatic Control, IEEE Transactions on, " vol. 40, no. 11, 1995, pp. $1943-1949$.

[6] Everdij, M. and Blom, H.," Embedding adaptive JLQG into LQ martingale control with a completely observable stochastic control matrix", Automatic Control, IEEE Transactions on, ”vol. 41, no. 3, 1996, pp. $424-430$.

[7] Xia, Y., Shi, P., Liu, G. and Rees, D.," Sliding mode control for stochastic jump systems with time-delay", in Intelligent Control and Automation, 2006.WCICA 2006," The Sixth World Congress on, vol. 1, 2006, pp. $354-358$.

[8] Wu, H.-N. and Cai, K.-Y.," Mode-independent robust stabilization for uncertain markovian jump nonlinear systems via fuzzy control”, Systems, Man ,and Cybernetics, Part B: Cybernetics, IEEE Transactions on, ”vol. 36, no. 3, 2005, pp. 509 -519. 
[9] Bo, W. and Haibo, J.," Robust $H_{\infty}$ control of uncertain stochastic nonlinear systems driven by noise of unknown covariance", in Control Conference,2008. CCC 2008. 27th Chinese," 2008, pp. $779-784$.

[10] Man, L., Zhao-jing, W., Wei, W. and Xiao-dong, D.," Adaptive output feedback control for stochastic nonlinear systems with zero dynamics", in Control and Decision Conference, 2009. CCDC '09. Chinese” 2009, pp. 1793 -1800.

[11] Hong, W., "Minimum entropy control of non-Gaussian dynamic stochastic systems", Automatic Control, IEEE Transactions on," vol. 47, no. 2, 2002, pp. 398 -403.

[12] Hong, W. and Yue, H., "Minimum entropy control of closed-loop tracking errors for dynamic stochastic systems", Automatic Control, IEEE Transactions on," vol. 48, no. 1, 2003, pp. $118-122$.

[13] Shannon. C., A mathematical theory of communication, The Bell System. Technical Journal, 1948.

[14] Pal, N. and. Pal, S., Entropy: a new definition and its applications", Systems, Man and Cybernetics, IEEE Transactions on," vol. 21, no. 5, 1991, pp. 1260 -1270.

[15] Silverman. B., Density Estimation for Statistics and Data Analysis, Chapman and Hall/CR, 1992.

[16] Yang, C. and Hong, W., "Minimum entropy control approach of closed-loop tracking errors for dynamic stochastic systems using yula parameterization formula", in Control Conference, 2006. CCC 2006. Chinese," 2006, pp. $1500-1505$.

[17] Zhang, J. and Hong, W., "Minimum entropy control of nonlinear arma systems over a communication network", Neural Computing and Applications," vol. 17, no. 4, 2008, pp. $385-390$.

[18] Afshar, P., Hong, W. and Chai, T., "An ILC-based adaptive control for general stochastic systems with strictly decreasing entropy”, Neural Networks, IEEE Transactions on, " vol. 20, no. 3, 2009, pp. $471-482$.

[19] Guo, L. and Hong, W., "Applying constrained nonlinear generalized PI strategy to PDF tracking control through square root B-spline models", Intentional Journal of Control," vol. 77, no. 17, 2004, pp. $1481-1492$.

[20] Owens, D. and Htnen, J., "Iterative learning control- an optimization paradigm", Annual Reviews in Control," vol. 29, no. 1, 2005, pp. $57-70$.

[21] Spong, M., "On the robust control of robot manipulators", Automatic Control, IEEE Transactions on," vol. 237, no. 11, 1992, pp. $1782-1786$.

[22] Shyu, K.-K., and Chu, P.-H. and Shang, L.-J., "Control of rigid robot manipulators via combination of adaptive sliding mode control and compensated inverse dynamics approach", Control Theory and Applications, IEE Proceedings, " vol. 143, no. 3, 1996, pp. $283-288$. 